\title{
Genome-Wide Pharmacogenomic Study of Neurocognition As an Indicator of Antipsychotic Treatment Response in Schizophrenia
}

\author{
Joseph L McClay*,', Daniel E Adkins', Karolina Åberg', Jozsef Bukszár', Amit N Khachane', \\ Richard SE Keefe ${ }^{2}$, Diana O Perkins ${ }^{3}$, Joseph P McEvoy ${ }^{2}$, T Scott Stroup ${ }^{4}$, Robert E Vann ${ }^{5}$, \\ Patrick M Beardsley, Jeffrey A Lieberman ${ }^{4}$, Patrick F Sullivan ${ }^{6,7}$ and Edwin JCG van den Oord' \\ 'Center for Biomarker Research and Personalized Medicine, School of Pharmacy, Medical College of Virginia Campus, Virginia Commonwealth \\ University, Richmond, VA, USA; ${ }^{2}$ Department of Psychiatry, Duke University, Durham, NC, USA; ${ }^{3}$ Department of Psychiatry, University of North \\ Carolina at Chapel Hill, Chapel Hill, NC, USA; ${ }^{4}$ Department of Psychiatry, Columbia University, New York, NY, USA; ${ }^{5}$ Department of \\ Pharmacology and Toxicology, Virginia Commonwealth University, Richmond, VA, USA; ${ }^{6}$ Departments of Genetics, Psychiatry, and Epidemiology, \\ University of North Carolina at Chapel Hill, Chapel Hill, NC, USA; 'Department of Medical Epidemiology and Biostatistics, Karolinska Institutet, \\ Stockholm, Sweden
}

\begin{abstract}
Neurocognitive deficits are a core feature of schizophrenia and, therefore, represent potentially critical outcome variables for assessing antipsychotic treatment response. We performed genome-wide association studies (GWAS) with 492K single nucleotide polymorphisms (SNPs) in a sample of 738 patients with schizophrenia from the Clinical Antipsychotic Trials of Intervention Effectiveness study. Outcome variables consisted of a neurocognitive battery administered at multiple time points over an I8-month period, measuring processing speed, verbal memory, vigilance, reasoning, and working memory domains. Genetic mediation of improvements in each of these five domains plus a composite neurocognitive measure was assessed for each of five antipsychotics (olanzapine, perphenazine, quetiapine, risperidone, and ziprasidone). Six SNPs achieved genome-wide significance using a pre-specified threshold that ensures, on average, only I in 10 findings is a false discovery. These six SNPs were located within, or in close proximity to, genes EHF, SLC26A9, DRD2, GPR I 37B, CHST8, and IL IA. The more robust findings, that is those significant across multiple neurocognitive domains and having adjacent SNPs showing evidence for association, were rs286913 at the EHF gene ( $p$-value $6.99 \times 10^{-8}, q$-value 0.034 , mediating the effects of ziprasidone on vigilance), rs I 1240594 at SLC26A9 ( $p$-value $1.4 \times 10^{-7}$, q-value 0.068 , mediating the effects of olanzapine on processing speed), and rs I I 6774 I 6 at IL IA ( $p$-value $6.67 \times 10^{-7}$, q-value $0.08 \mathrm{I}$, mediating the effects of olanzapine on working memory). This study has generated several novel candidate genes for antipsychotic response. However, our findings will require replication and functional validation. To facilitate replication efforts, we provide all GWAS p-values for download.
\end{abstract}

Neuropsychopharmacology (20II) 36, 616-626; doi:I0.1038/npp.2010.193; published online 24 November 2010

Keywords: genome-wide association; schizophrenia; pharmacogenomics; personalized medicine; single nucleotide polymorphisms

\section{INTRODUCTION}

Schizophrenia is an often devastating neuropsychiatric illness. Biological factors have been strongly implicated and considerable efforts are currently being devoted to finding genes that increase susceptibility to the disease. One

*Correspondence: Dr LL McClay, Center for Biomarker Research and Personalized Medicine, Virginia Commonwealth University, McGuire Hall, Medical College of Virginia Campus, III2 East Clay Street, Richmond, VA 23298-0533, USA, Tel: + I 804828 3428, Fax: + I 804 628 399।, E-mail: jlmcclay@vcu.edu

Received 4 March 2010; revised 16 September 2010; accepted 23 September 2010 eventual goal of these efforts is the identification of novel drug targets to develop better therapeutic compounds (Sanger, 2004; Williams, 2003). However, the development of new drugs is extremely expensive and takes many years (Roses, 2000). Arguably, a more immediate role for genetic markers in the treatment and management of schizophrenia is to improve our ability to tailor the prescription of existing drugs to individual patients (Malhotra et al, 2004). Typically, only a proportion of patients respond to any given antipsychotic drug (Kane, 1999). The several weeks it may take clinicians to declare a treatment ineffective leaves the patient vulnerable to continuing dysfunction and suicide (Meltzer and Okayli, 1995). The clinical benefits of 
a genetic test to identify the most effective antipsychotic treatment for each patient at time of first presentation would be considerable (Basile et al, 2002).

Despite some encouraging signs, efforts to predict antipsychotic treatment response have met with limited success (Malhotra et al, 2004). One of the complications is that neuropsychiatric clinical phenotypes are complex and heterogeneous and, therefore, may not be optimal measures in pharmacogenomic studies. An alternative is to focus on the amelioration of specific deficits related to the disorder. Patients with schizophrenia show deficits across a large number of neurocognitive domains and these deficits are regarded as the single strongest correlate of real world functioning (Green, 1996). Although there is variability among patients (Kremen et al, 2004), neurocognitive deficits are present by the first episode and persist throughout the course of the illness (Mesholam-Gately et al, 2009). Substantial evidence points to neurocognitive deficits in the relatives of schizophrenic probands (Hill et al, 2008) and a large consortium study found moderate, but significant, heritabilities for all neurocognitive measures investigated, with estimates ranging from 24 to $55 \%$ (Greenwood et al, 2007). The motivational and cognitive impairments of schizophrenia are strongly associated with the long-term disability typically produced by the disease, and this is why cognitive deficits are increasingly being seen as targets for treatment (Hyman and Fenton, 2003). Crucially, for our purposes, previous studies have shown improvement in neurocognitive function following treatment with several different antipsychotic drugs (Keefe et al, 2007a,b). Taken together, these observations suggest that neurocognitive deficits represent potentially critical outcome variables for the pharmacogenomic study of antipsychotic drug response.

To date, pharmacogenomics studies have typically focused on candidate genes, selected for encoding of drug targets (pharmacodynamic candidates) or for involvement in the metabolism of the drug itself (pharmacokinetic candidates). Pharmacodynamic candidates in schizophrenia include dopamine or serotonin receptors and several studies have shown positive associations between, for example, dopamine receptors D2 (DRD2) and D3 (DRD3) and antipsychotic response (see Arranz and de Leon, 2007, for a review). Pharmacokinetic candidates, particularly the cytochrome P450 genes, are well known to harbor genetic variation affecting the metabolism of antipsychotics (Kirchheiner et al, 2004). Nevertheless, a limitation of prior studies in this area is that the selection of candidate genes is restricted to current knowledge about mechanisms of drug action. More exploratory methods that systematically screen the whole genome for association with drug response likely represent a superior strategy for discovering relevant genetic variation. Such genome-wide association studies (GWAS) have become feasible in recent years and have already shown considerable promise to identify novel associations between genetic variants and complex traits (Altshuler et al, 2008). Indeed, several large-scale GWAS for schizophrenia have been recently published. Associations have been found with, for example, the MHC region (Shi et al, 2009) and novel loci such as ZNF804A (O'Donovan et al, 2008). To date, though, the number of GWAS on antipsychotic response is considerably more modest (Zandi and Judy, 2010).
In this study, we use the Clinical Antipsychotic Trials of Intervention Effectiveness (CATIE) study (Lieberman et al, 2005; Stroup et al, 2003) to detect genetic variation underlying changes in neurocognitive functioning following treatment with five different antipsychotics. The sample consisted of 738 CATIE subjects with DSM-IV schizophrenia diagnoses who were genotyped using the Affymetrix $500 \mathrm{~K}$ platform plus a custom $164 \mathrm{~K}$ chip to improve genomewide coverage (Sullivan et al, 2008). Treatment outcome was measured using a comprehensive neurocognitive assessment battery in five domains: processing speed, verbal memory, vigilance, reasoning, and working memory, in addition to a composite measure that condensed information from these five sub-domains (Keefe et al, 2003, 2006).

\section{MATERIALS AND METHODS}

\section{Study Sample}

A total of 765 subjects from the CATIE study gave informed consent to participate in genetic studies. The patients were diagnosed with schizophrenia using the Structured Clinical Interview for DSM-IV (First et al, 1994). This sample has been carefully described elsewhere (Lieberman et al, 2005; Stroup et al, 2003). In short, CATIE is a multiphase randomized controlled trial of antipsychotic medications, including the second-generation drugs, olanzapine, quetiapine, risperidone, and ziprasidone, compared with a midpotency first-generation drug, perphenazine. Patients were followed for up to 18 months and, to maximize representativeness, the participants were recruited from 57 clinical settings around the United States. The mean age for the participants was 40.9 years and on average they first received antipsychotic medication 14.3 years previously. All participants or their legal guardians gave written informed consent and the institutional review board at each site approved the study.

\section{Neurocognitive Assessment Battery}

For CATIE, a neurocognitive advisory group was convened and arrived at consensus regarding the contents, standardization, and methodology of the neurocognitive assessment battery (Keefe et al, 2003, 2006). Eleven tests were administered, resulting in 24 individual scores. Factor analysis showed that a model comprised of five domain scores provided the best fit (Keefe et al, 2006). The five neurocognitive domains were processing speed that is based on the standardized mean of grooved pegboard (Lafayette Instrument Company, 1989), WAIS-R digit symbol test (Wechsler, 1981), and the mean of the two verbal fluency measures (Benton, 1978); verbal memory was assessed with the Hopkins verbal learning test (average of three trials) (Brandt, 1991); vigilance was based on the continuous performance test d-prime scores (average of 2-digit, 3-digit, and 4-digit) (Cornblatt et al, 1988); reasoning summary score was the mean of Wisconsin card sorting test (Heaton et al, 1993) and WISC-R mazes (Wechsler, 1991); working memory summary score was the average of a computerized test of visuospatial working memory (sign reversed) (Lyons-Warren et al, 2004) and letter number sequencing 
Table I Number of Subjects/Assessments Analyzed for Each Antipsychotic and Neurocognitive Outcome

\begin{tabular}{|c|c|c|c|c|c|}
\hline & Olanzapine & Perphenazine & Quetiapine & Risperidone & Ziprasidone \\
\hline Verbal memory & $223 / 459$ & $109 / 223$ & 198/325 & $213 / 413$ & | 38/254 \\
\hline Vigilance & $215 / 430$ & $102 / 203$ & 190/305 & $202 / 381$ & 133/234 \\
\hline Processing speed & $223 / 459$ & $109 / 223$ & 198/325 & $213 / 413$ & $138 / 254$ \\
\hline Reasoning & $223 / 459$ & $109 / 223$ & 198/325 & $213 / 413$ & $138 / 254$ \\
\hline Working memory & $223 / 459$ & $108 / 222$ & 198/325 & $213 / 413$ & $138 / 254$ \\
\hline
\end{tabular}

The slightly smaller numbers in vigilance are attributable to invalid scores from computerized testing (see Keefe et al, 2006).

(Gold et al, 1997). The correlations among the five domain scores were medium to high and we, therefore, also analyze the standardized composite score of these five domains (Keefe et al, 2006).

\section{Estimating Treatment Effects}

Multiple assessments were available per patient (Table 1). There was a mean of 3.4 assessments per subject. Conventional approaches to calculating treatment effects (eg, post-treatment-pre-treatment difference scores) define treatment using only two observations. Alternatively, using all available assessments can substantially improve statistical power to detect true associations. For this reason, we have developed a systematic method (van den Oord et al, 2009) using mixed effects modeling (Goldstein, 1995; Searle et al, 1992) to estimate treatment effects. We first determine the functional form of the drug response trajectory, then screen many possible covariates to select those that improve the precision of the treatment effect estimates, and finally generate individual drug effect estimates based on the best fitting model using best linear unbiased predictors (Pinheiro and Bates, 2000) (see Supplementary Material for details). Intuitively, these treatment effects quantify how much each subject's phenotype changes in response to a given drug, relative to the average effect for all subjects who took the drug. Each treatment effect measure was screened for outliers using the multivariate outlier method developed by Hadi (Hadi and Simonoff, 1993). This procedure quantifies the (Mahalanobis) distance of each observation from the centroid of all observations. We excluded observations that were outlying at $p<0.05$. The majority of distributions showed 0 outliers and none exhibited $>2$ (7 total outliers out of 30 outcome distributions). After excluding outliers, outcomes were all virtually perfectly normally distributed: mean skewness $=-0.025$ or 0.209 when taking the mean of the absolute values, while mean kurtosis $=0.534$ (signed) or 0.585 (absolute).

\section{Genotyping and Genotype Quality Control}

DNA sampling, GWAS genotyping, and genotype quality control (QC) have been described previously for this sample (Sullivan et al, 2008). Briefly, a total of 665439 single nucleotide polymorphisms (SNPs) were genotyped for each subject using both the Affymetrix $500 \mathrm{~K}$ ' $\mathrm{A}$ ' chipset (Santa Clara, CA, USA) and a custom 164K SNP-chip created by Perlegen (Mountain View, CA, USA). Highly stringent QC protocols were implemented. Of the 500568 SNPs on the
Affymetrix arrays, $79.8 \%$ passed initial QC, while $66.1 \%$ of the 164871 SNPs on the custom Perlegen chip passed initial QC. Additional SNPs with allele frequencies $<0.01$ and those with 2 disagreements among 36 pairs of replicate samples were removed. Subjects with sex discrepancies, cryptic duplicates, and those with excessive missing genotypes were removed (see Supplementary Materials). After QC, a total of 492900 SNP genotypes from 738 individuals remained for analysis.

\section{Ancestral Background}

Approximately $57 \%$ of the CATIE subjects self-identify themselves as white/European American (EA) and 29\% as black/African American (AA). The remaining 14\% of the patients consider themselves to have 'other' ancestral origins or to belong to multiple ancestral categories. To avoid false positives due to population stratification, Sullivan et al (2008) previously performed an extensive evaluation of multiple methods to control for ancestral heterogeneity in CATIE. They concluded that the principal component and multi-dimensional scaling (MDS) approach worked best. We, therefore, proceeded with the MDS approach as implemented in PLINK (Purcell et al, 2007). For our analyses, we used five MDS dimensions that appeared to capture the majority of ancestry-related effects in CATIE (see Supplementary Materials for details).

\section{Association Testing and False Discovery Rate Control}

All association testing was conducted in PLINK (Purcell et al, 2007) using a linear regression model with the five population stratification MDS dimensions as covariates. We used a false discovery rate (FDR) (Benjamini and Hochberg, 1995)-based approach to declare significance. In comparison with controlling a family-wise error rate (eg, Bonferroni's correction), the FDR (a) provides a better balance between finding true effects $v s$ controlling false discoveries, (b) results in comparable standards for declaring significance across studies because it does not directly depend on the number of tests, and (c) is relatively robust against having correlated tests (Brown and Russell, 1997). The FDR is commonly used in many high-dimensional applications and has also successfully been applied in the context of GWAS (Beecham et al, 2009; Lei et al, 2009; Liu et al, 2009). As motivated previously, we chose an FDR threshold of 0.1 for declaring genome-wide significance (van den Oord and Sullivan, 2003). This means that on average $10 \%$ of the SNPs declared significant are expected to be false discoveries. 
Operationally (Black, 2004), the FDR was controlled using $q$-values that are FDRs calculated using the $p$-value of the markers as thresholds for declaring significance (Storey, 2003; Storey and Tibshirani, 2003). It is important to note that performing many GWAS analyses does not present a problem for the FDR because it controls the expected ratio of false to all discoveries. Thus, when many GWAS are performed, the number of false positives will increase and so will the number of true positives. The expected ratio of false to all discoveries will, therefore, remain 0.1 with our threshold for declaring genome-wide significance regardless of how many GWAS are performed.

To avoid an all-or-nothing conclusion about whether an SNP is significant and improve the interpretation of our GWAS results, we also estimated for each SNP the local FDR (lFDR) (Efron et al, 2001) using a precise method specifically designed for GWAS studies (Bukszar et al, 2009). This estimated $l$ FDR is the posterior probability that the SNP has no effect. In addition to reporting $p$ - and $q$-values, we also report the effect size for our top findings, that is increase in explained variance $\left(r^{2}\right)$ for each of the genome-wide significant SNPs when the SNP in question is added to the regression model (without the MDS dimensions).

\section{Candidate Gene Analysis}

To select candidate genes for drug effects, we selected all genes that had previously shown positive associations with response to any antipsychotic drug using any outcome measure, as reviewed by Arranz and de Leon (2007), plus additional genes with reported positive associations since publication of that article (Anttila et al, 2007; Campbell et al, 2008; Hamdani et al, 2008; Hwang et al, 2007; Mancama et al, 2007; Meary et al, 2008). We also included all genes involved in the major and secondary metabolic pathways of antipsychotic drugs (Arranz and de Leon, 2007). Finally, we also included two genes (RIMS1 and GRM8) that showed promising associations in a recent candidate gene study of antipsychotic response in CATIE (Need et al, 2009), using a different analytical approach to that presented here. This gave a total of 41 candidate genes. SNPs were selected in these genes using a liberal definition of the gene boundary ( $\pm \sim 50 \mathrm{~kb})$, to avoid excluding SNPs in the region that may be in linkage disequilibrium (LD) with others within the gene itself, or that may be in proximal regions of regulatory importance. In total, we identified 1595 SNPs corresponding to the 41 candidate genes - 39 SNPs per gene (see Supplementary Table S4; Supplementary Material).

\section{RESULTS}

When generating the outcome measures, two were found to show limited individual differences in treatment response, that is variance was close to zero. These were perphenazine with vigilance and perphenazine with working memory. GWAS were not performed for these outcomes because any findings would not be associated with individual differences in treatment, but simply sampling error. Quantile-Quantile (QQ) plots and Manhattan plots for each of the remaining successful GWAS are available in the online Supplementary
Table 2 Number of GWAS q-values Below Various Thresholds

\begin{tabular}{lrrrrr}
\hline & \multicolumn{5}{c}{ q-value threshold } \\
\cline { 2 - 6 } & $\mathbf{0 . 1}$ & $\mathbf{0 . 2 5}$ & $\mathbf{0 . 5}$ & $\mathbf{0 . 7 5}$ & $\mathbf{0 . 9 5}$ \\
\hline By neurocognitive scale & & & & & \\
Neurocognitive composite & 0 & 0 & 3 & 78 & 160 \\
Working memory & 4 & 12 & 43 & 95 & 162 \\
Reasoning & 0 & 10 & 27 & 49 & 171 \\
Processing speed & 1 & 1 & 8 & 61 & 97 \\
Verbal memory & 0 & 0 & 2 & 29 & 126 \\
Vigilance & 1 & 2 & 4 & 8 & 97 \\
Total & 6 & 25 & 87 & 320 & 813 \\
& & & & & \\
By drug & & & & & \\
Olanzapine & 5 & 10 & 23 & 32 & 141 \\
Quetiapine & 0 & 11 & 48 & 206 & 286 \\
Risperidone & 0 & 2 & 9 & 31 & 184 \\
Ziprasidone & 1 & 2 & 5 & 29 & 141 \\
Perphenazine & 0 & 0 & 2 & 22 & 61 \\
Total & 6 & 25 & 87 & 320 & 813 \\
\hline & & & & &
\end{tabular}

Material. All $p$-values for each GWAS are available for download at http://www.people.vcu.edu/ jlmcclay. Table 2 shows the number of significant GWAS results at various FDR thresholds. For concision, Table 2 presents significant results grouped by outcome or by drug. Six SNPs exceeded our pre-specified genome-wide significance threshold, which controls the FDR at the 0.1 level (van den Oord and Sullivan, 2003). When grouped by medication, the largest numbers of genome-wide significant results were found for olanzapine $(m=5)$; when grouped by neurocognitive outcome, the largest number of findings were found for working memory $(m=4)$. Figure 1 shows the regional plots of the genome-wide significant findings, while Figure 2 shows the outcome scores by genotype.

Table 3 provides details on those SNPs that were either genome-wide significant $(q$-value $<0.1)$ or 'potentially interesting' at a $q$-value threshold $<0.25$. The most significant SNP overall, rs286913, reached genomewide significance $\left(p\right.$-value $=6.99 \times 10^{-8}, q$-value $=0.034$, $\left.r^{2}=0.213\right)$ and appeared to mediate the effects of ziprasidone on vigilance. This SNP lies within an intron of the $E H F$ (ETS homologous factor) gene that is located on chromosome 11p12. An additional SNP in EHF, rs286928, also mediated the effects of ziprasidone on vigilance and was located some $16 \mathrm{~kb}$ from rs286913. LD between these two markers was high, with $r^{2}=0.87$ in EAs and 0.46 in AAs. A regional plot of $p$-values for all CATIE SNPs at this locus (Figure 1) shows their position relative to the gene. Haplotype analysis of these two SNPs plus an additional six flanking markers yielded several highly significant findings in the EA subsample (the largest single ethnic group in CATIE). However, the best $p$-value among the haplotypes tested $\left(p=1.18 \times 10^{-5}\right)$ was only marginally more significant than the single marker $p$-value for rs 286913 in EA $\left(p=1.8 \times 10^{-5}\right)$, suggesting that this marker alone is 

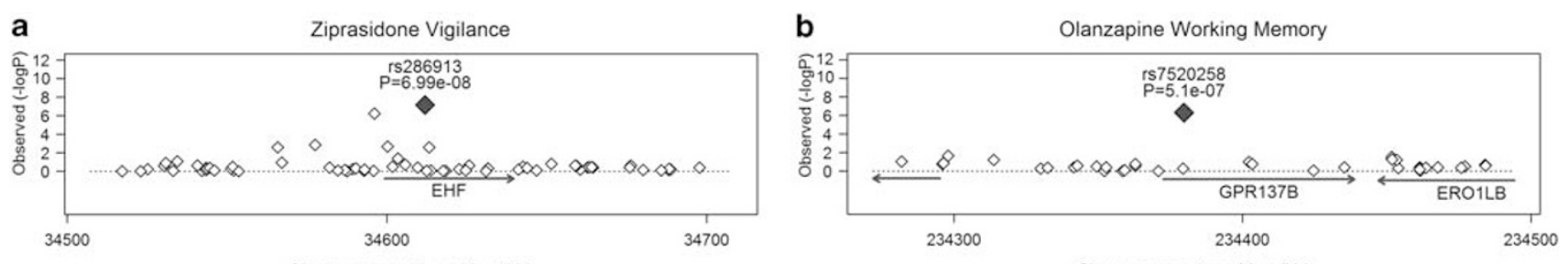

Chromosome 11 position $(\mathrm{kb})$
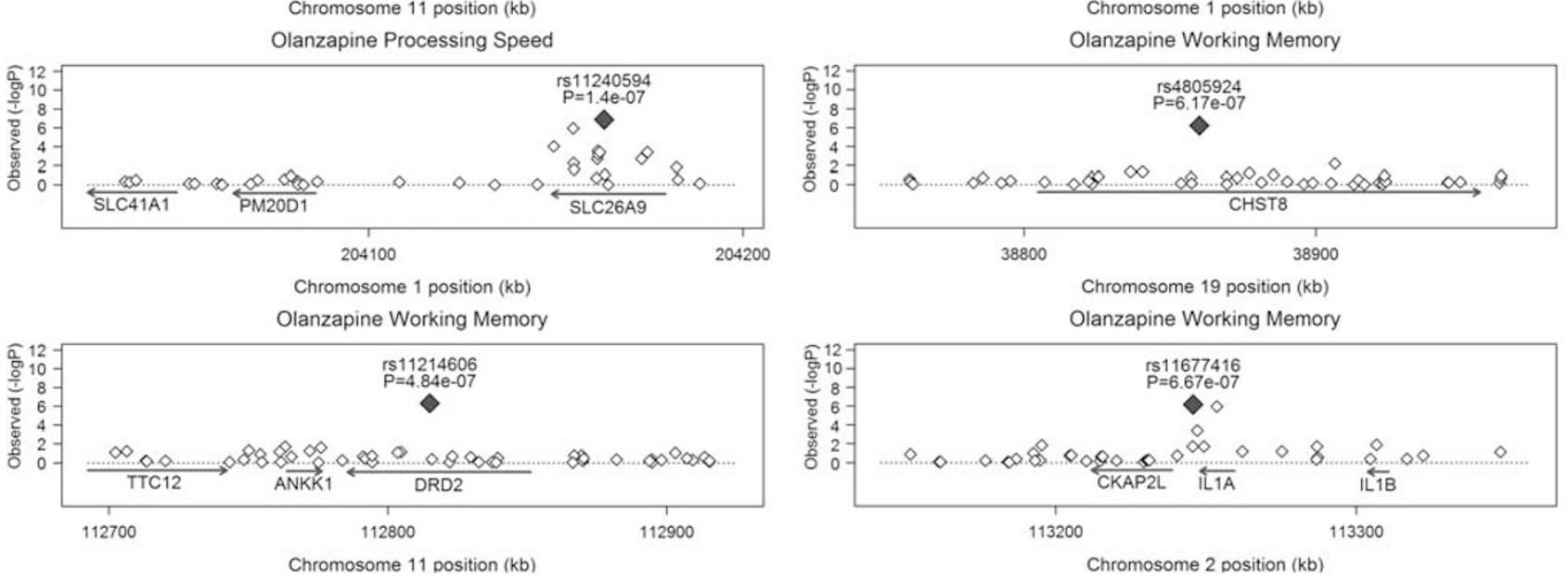

Figure I ( $a$ and b) Regional plots of $p$-values for SNPs flanking the six genome-wide significant findings (solid black dots). The $y$ axis is the -log 10 of the $p$-values for each SNP, while the $x$ axis denotes genomic position according to genome build 36 (hgl8). All genotyped SNPs within $\sim \pm 100 \mathrm{~kb}$ of the primary findings are plotted.

rs286913

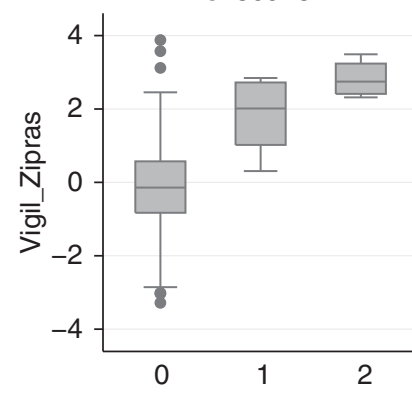

rs11677416

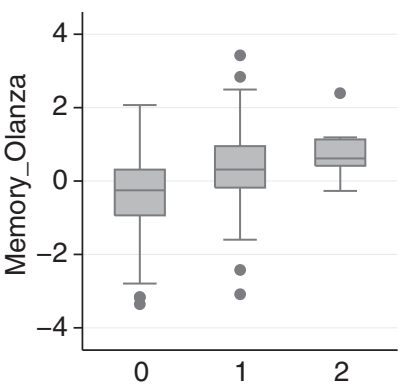

rs11240594

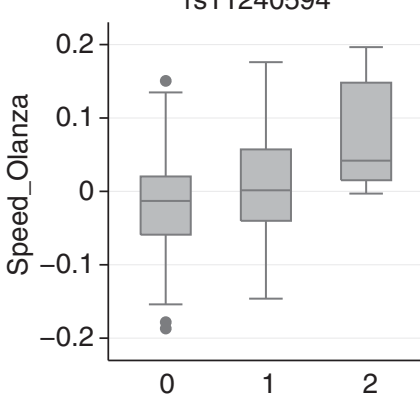

rs4805924

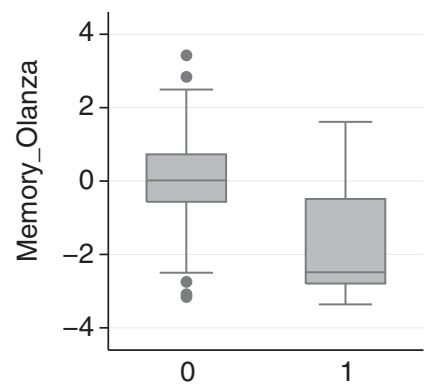

rs7520258

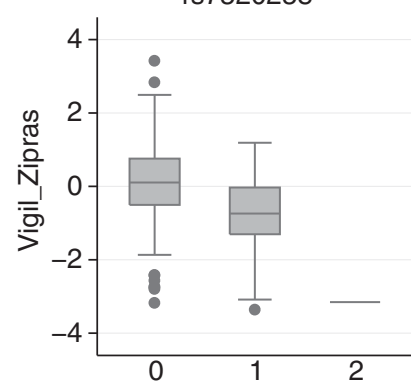

rs11214606

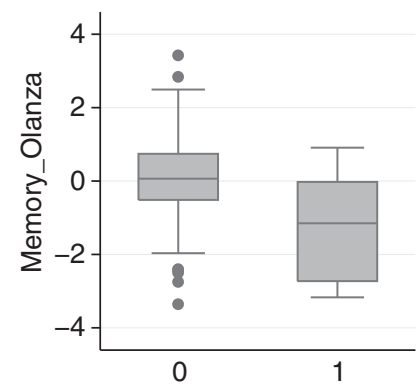

Figure 2 Box and whisker plots of treatment response, by genotype, for the six genome-wide significant SNP associations. The $y$ axis units are SD. Vigil_zipras=vigilance with ziprasidone; Speed_olanza=processing speed with olanzapine; Memory_olanza= working memory with olanzapine. For rs4805924 and rs I 1214606, only two genotpype groups are plotted. Although rare allele homozygotes were present for both SNPs in CATIE, none of these had at least two observations in the relevant neurocognitive outcome/drug combination (working memory and olanzapine) to allow a treatment effect to be calculated.

probably sufficient to capture the association. In addition to mediating the effects of ziprasidone on vigilance, rs286913 showed association $(p<0.05)$ for additional drug and outcome combinations. These included ziprasidone with the neurocognitive composite measure $(p=0.0082)$, risperidone and reasoning $(p=0.0015)$, and risperidone with working memory $(p=0.035)$. The $p$-values for all six genome-wide significant SNPs across all 28 drug/outcome combinations are shown in Supplementary Tables S4A and $\mathrm{B}$ in the Supplementary Material. 
Table 3 Summary of top findings with q-values below 0.25.

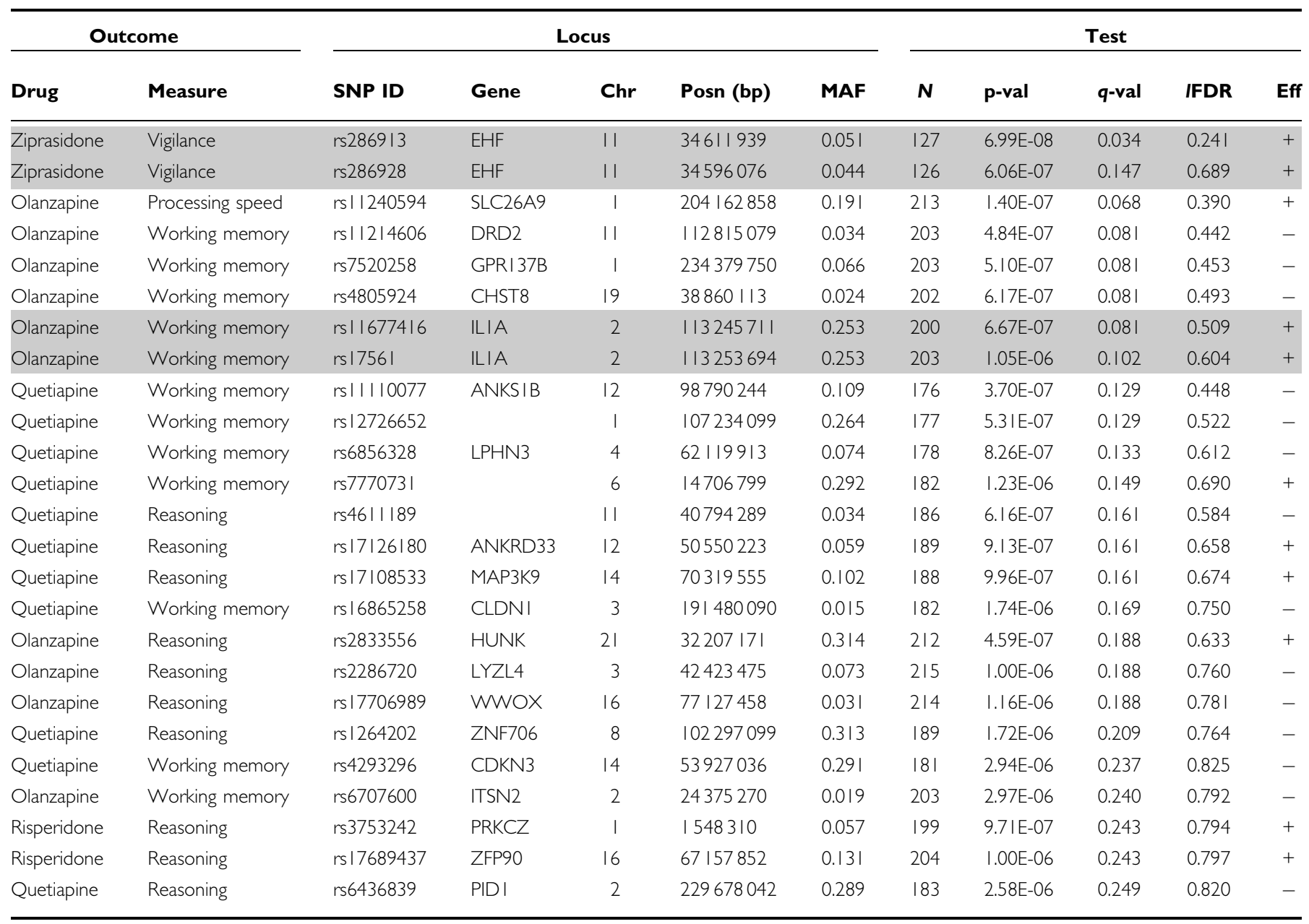

Summary of top findings with $q$-values below 0.25 . Where more than one marker at a locus has findings $q<0.25$, these have been grouped and shaded in grayscale. Genomic position is indicated according to genome build 36 (hg /8). MAF is the minor allele frequency and IFDR is the local false discovery rate, which provides a marker-specific estimate of the posterior probability that the finding is false positive. 'Eff' is the direction of effect of the minor allele, eg, '+ ' indicates a greater improvement in neurocognitive function for the minor allele compared with the major allele.

The second most significant locus was rs11240594 on chromosome 1q32 that appeared to mediate the effects of olanzapine on processing speed $\left(p\right.$-value $=1.4 \times 10^{-7}$, $q$-value $\left.=0.068, r^{2}=0.125\right)$. The SNP is located in an intron of SLC26A9 (solute carrier family 26 (sulfate transporter), member 9). Multiple neighboring SNPs in LD with rs11240594 also appeared to tag the same signal (see Figure 1). These included rs12727528 ( $p$-value $=5.65 \times 10^{-5}$ in EA) and several other markers with $p$-values $\sim 10^{-3}$. In addition to mediating the effects of olanzapine on processing speed, rs11240594 showed association $(p<0.05)$ with olanzapine and verbal memory $(p=0.0377)$, vigilance $(p=0.0434)$, and the neurocognitive composite $(p=0.0022)$, in addition to ziprasidone with processing speed $(p=0.0012)$.

Among the six genome-wide significant results was rs11214606 at $D R D 2$ that mediated the effect of olanzapine on working memory ( $p$-value $4.84 \times 10^{-7}, q$-value 0.081 , $\left.r^{2}=0.120\right)$. As DRD2 is a well-known candidate for antipsychotic response and a drug target of all antipsychotic medications, this was an encouraging finding. Nevertheless, as can be seen in Figure 1, rs11214606 is an orphan finding, with no other SNPs in the region showing substantial evidence for association. Consistent with HapMap data, however, LD between rs11214606 and neighboring markers is very low, that is $r^{2}$ does not exceed 0.05 in the CATIE sample. Therefore, we cannot expect neighboring markers to tag the same signal. SNP rs11214606 did not show association at the $p<0.05$ level with any other drug or outcome combination, although there were trends approaching significance with other outcomes related to olanzapine (see Supplementary Material).

All of the remaining SNPs achieving genomewide significance were also with olanzapine and working memory. These included rs7520258 ( $p$-value $5.1 \times 10^{-7}, q$-value $0.081, r^{2}=0.120$ ), located in an intron of the GPR137B (G protein-coupled receptor 137B) gene on chromosome 1q42-43 and rs4805924 ( $p$-value $6.1 \times 10^{-7}$, $q$-value $\left.0.08, r^{2}=0.119\right)$, which is situated $\sim 7 \mathrm{~kb}$ from the start of transcription at CHST8 (carbohydrate ( $N$-acetylgalactosamine 4-0) sulfotransferase 8) on chromosome 19q13. (However, the more recent genome build 37.1 provisionally locates rs4805924 in an intron of CHST8). As can be seen 
from the regional plots in Figure 1, both of these findings are isolated with no neighboring SNPs showing robust evidence for association. In particular, it is worth remarking that rs7520258 at GPR137B is almost invariant in EAs (MAF $<0.01$ in CATIE), suggesting that the signal, if true, is almost entirely coming from the non-EA ethnic groups. For the finding at CHST8, HapMap shows rs4805924 to be in an LD block of $22 \mathrm{~kb}$. However, within this block only two HapMap SNPs were in high LD with rs4805924 (rs16968363 and rs10500266, both with $r^{2}=0.79$ ) and these were not genotyped in CATIE. Of the remaining SNPs in the block that were genotyped in our sample, none showed LD with $r^{2}>0.15$ and, therefore, could not be expected to tag the same signal. Nevertheless, despite a lack of suitable SNP proxies, both of these genome-wide significant SNPs showed some evidence for association $(p<0.05)$ across different neurocognitive domains and drugs. In addition to the primary finding with olanzapine and working memory, rs7520258 appeared to mediate the effects of ziprasidone on processing speed $(p=0.0133)$, while rs4805924 showed association with olanzapine and processing speed $(p=0.0045)$, in addition to olanzapine and the neurocognitive composite $(p=0.0029)$.

The final genome-wide significant finding, again with olanzapine and working memory, is rs11677416 ( $p$-value $6.67 \times 10^{-7}, q$-value $\left.0.081, r^{2}=0.119\right)$, located $\sim 2 \mathrm{~kb}$ from the boundary of IL1A (interleukin-1- $\alpha$ ) on chromosome 2q14. This SNP showed evidence for association across other neurocognitive domains, including olanzapine with reasoning $(p=0.0045)$, vigilance $(p=0.0084)$, and the neurocognitive composite $(p=0.0003)$, in addition to perphenazine and reasoning $(p=0.0065)$. Another SNP, rs17561, $\sim 8 \mathrm{~kb}$ from rs11677416, was almost genome-wide significant with a $q$-value of 0.102 . This latter SNP is located in the coding sequence of $I L 1 \mathrm{~A}$ and gives rise to a nonsynonymous amino-acid substitution of alanine to serine at protein position 114. Consistent with HapMap data, the two SNPs were in almost perfect LD in our sample $\left(r^{2}=0.97\right.$ in AA and 0.99 in EA), suggesting that both markers are tagging the same signal.

\section{Candidate Gene Analysis}

In addition to the GWAS results, we also report our findings for markers located in, or in the vicinity of, specific candidate genes. Examination of 1595 SNPs at 41 candidate genes, that is $\sim 39$ SNPs per gene (see Supplementary Table S5; Supplementary Material, p15) yielded only three findings with $q$-values below 0.5 . The remaining tests all had $q$-values very close to 1 . This was somewhat surprising, because far fewer tests are performed relative to GWAS and FDR-based $q$-values are known to be too liberal in these scenarios (Zaykin et al, 2000). However, the lack of complete coverage for many important candidate genes using these arrays, as we have discussed before (McClay et al, 2009), means that we cannot reliably exclude association for many of these loci. The top finding in this analysis was rs11214606 at $D R D 2$, mediating the effects of olanzapine on working memory $\left(p\right.$-value $=4.84 \times 10^{-7}$, $q$-value $=0.022)$. This is the same SNP at DRD2 that was genome-wide significant in the main analysis. The remaining findings with 'potentially interesting' $q$-values were rs7766029 at cannabinoid receptor 1 (CNR1), mediating the effects of perphenazine on verbal memory $\left(p\right.$-value $=1.76 \times 10^{-5}, q$-value $\left.=0.391\right)$ and $\mathrm{rs} 936465$ at DRD4 that mediated the effects of ziprasidone on the neurocognitive composite measure $\left(p\right.$-value $=3.13 \times 10^{-5}$, $q$-value $=0.463)$.

\section{DISCUSSION}

Understanding the genetic variation affecting response to antipsychotic drugs is important to develop novel diagnostic tests to match individual patients with schizophrenia to the most effective and safe medication. In this study, we performed GWAS of antipsychotic response, using neurocognitive function as the outcome variable(s). In addition to being a core deficit in schizophrenia, neurocognitive functions can be viewed as endophenotypes for the disorder and as such provide outcome measures that are potentially more genetically simple and tractable. In total, six markers achieved genome-wide significance in our study. In comparison with a previous study that we carried out in CATIE that used schizophrenia symptoms on the positive and negative syndrome scale (PANSS) as the outcome variables, this study with neurocognitive phenotypes would appear to be the more successful. Our study using the PANSS found only a single SNP to be significant at a genome-wide level and only three SNPs in genes with $q$-values $<0.5$ (McClay et al, 2009).

An SNP in DRD2 reached genome-wide significance, mediating the effect of olanzapine on working memory. $D R D 2$ is a well-known candidate for antipsychotic response and is a drug target of olanzapine (Kapur et al, 1999). However, despite the strong theoretical rationale for this gene being associated with antipsychotic drug response, our association evidence for $D R D 2$ is perhaps less robust than is the case with some of the other genome-wide significant findings. First, no neighboring SNPs in our sample showed evidence for association, although LD between neighboring markers was low. Furthermore, the SNP was not significantly associated with other outcome variables in our study, although there were trends toward significance with some other olanzapine-related variables that were in the same direction as the primary finding (eg, reasoning, neurocognitive composite - see Supplementary Table S4A in the Supplementary Material). If the effect is real, it is likely quite small and, therefore, difficult to detect consistently in samples of the size used here. Even in view of DRD2's status as a likely antipsychotic pharmacogene, our finding at this locus should still be viewed as preliminary and requiring replication.

Our top finding overall was an SNP in the EHF (ETS homologous factor) gene on chromosome 11 . This gene is a member of the ETS family of transcription factors, which are involved in many aspects of gene regulation and development. EHF is expressed at high levels in epithelial tissues, primarily in glandular epithelium such as prostate, pancreas, salivary gland, and trachea (Kas et al, 2000). Out of 438 EHF tissue-specific human cDNA clones in AceView (Thierry-Mieg and Thierry-Mieg, 2006) (http://www.ncbi. nlm.nih.gov/IEB/Research/Acembly/index.html), only a single clone of $E H F$ was found in brain. Thus, while expression in brain cannot be completely ruled out, it is likely to be at 
very low levels. Current information on this gene and its function is relatively sparse. One of the few specific known actions of $E H F$ is as a transcriptional repressor downstream of mitogen-activated protein kinase (MAPK) signaling cascades (Tugores et al, 2001). This is possibly of note because mitogen-activated protein kinase kinase kinase 9 (MAP3K9), an epithelial-specific MAPK (Dorow et al, 1993), also emerged as one of our most significant findings (Table 3).

The second best genome-wide significant finding was with SLC26A9 (solute carrier family 26, sulfate transporter, member 9), which is a member of the SLC26 sulfate/anion transporter gene family. These are anionic transporters involved in chloride and bicarbonate ion absorption or secretion in epithelia (Loriol et al, 2008). The product of SLC26A9 is a highly selective chloride ion channel that is regulated by WNK kinases (Dorwart et al, 2007). It is expressed at high levels in airway and gastric surface epithelia, but is also expressed in the brain (based on the AceView (Thierry-Mieg and Thierry-Mieg, 2006) and UniGene (Wheeler et al, 2003) databases). It is a relatively uncharacterized gene, but its regulation by WNK kinases, specifically WNK4, may be significant. WNK4 has been shown to interact with intersectin (He et al, 2007) and we found ITSN2 (intersectin 2) among our top findings $(q$-value $=0.24)$. WNK4 is also known to regulate cellular chloride ion permeability and is localized to tight junctions (Kahle et al, 2004), that is networks of sealing strands that join epithelial cell membranes, thereby creating a virtually impermeable barrier to fluid. One of the two major classes of tight junction proteins is the claudins, the other being the occludens. We found CLDN1 (claudin 1) to be associated with quetiapine and working memory with a $q$-value of 0.169. Claudins are well recognized to have a major function in cellular permeability (Tsukita and Furuse, 2000).

These associations with EHF and other epithelial-related genes would not appear to have such an obvious connection to antipsychotics as compared, for example, with DRD2. However, with the caveat that these findings still require replication, a plausible hypothesis is that these genes are involved in epithelial barrier permeability. The role of epithelial barriers in pharmacokinetics has long been established. As such, our GWAS may have simply uncovered specific loci harboring variation that contribute to individual differences in barrier permeability, and by extension, antipsychotic availability and efficacy. As most antipsychotics are administered orally, absorption across gut epithelia is a plausible mechanism, as is elimination of drug across hepatic or renal epithelia. Also, it is conceivable that the closely related endothelial tissues of the bloodbrain barrier may be involved.

Two SNPs among our top hits were at interleukin-1- $\alpha$ (IL1A), which also appeared to mediate the effect of olanzapine on working memory. It has been shown that intravenous administration of human interleukin-1- $\alpha$ protein induces memory impairments in mice (Banks et al, 2001). However, there does not appear to be any existing evidence in the literature linking $I L 1 A$ to antipsychotics or schizophrenia. Interleukin-1- $\beta$ (IL1B), which is located $\sim 45 \mathrm{~kb}$ from IL1A, has been previously associated with altered brain structure in patients with schizophrenia (Meisenzahl et al, 2001). However, the association in this study is clearly localized to $I L 1 A$ (Figure 1) and as mentioned in Results, one of the two top SNPs in IL1A (rs17561) is non-synonymous, leading to an amino-acid change at protein position 114. This SNP, therefore, makes an appealing target for future functional study.

We previously reported $A N K S 1 B$ as one of our top findings in a CATIE pharmacogenomics GWAS, mediating the effects of olanzapine on negative symptoms (McClay et al, 2009), as measured by the PANSS. In this study, we again find $A N K S 1 B$ among our top findings, this time mediating the effect of quetiapine on working memory. As these findings are both in the same sample, and negative symptoms are known to be correlated with neurocognitive functioning, the fact that we see the same gene appearing in both analyses may not appear too surprising. However, in CATIE, the subjects being treated with one drug are for an important part different from those treated with a second drug. For example, only 49 CATIE subjects received both quetiapine and olanzapine. Furthermore, correlations between drug effects on negative symptoms and cognitive function (working memory in this specific instance) are present, but not particularly large (typically in the $0.1-0.2$ range). Thus, due to largely non-overlapping samples and modest correlation between drug effects on negative symptoms and cognitive function, the $A N K S 1 B$ findings in CATIE involve to a large extent independent observations. Furthermore, it is of note that $A N K S 1 B$ also appeared among the top findings in a GWAS of citalopram response carried out by another group in the sequenced treatment alternatives to relieve depression (STAR*D) sample (Garriock et al, 2010). While this gene remains to be fully characterized, a recent study identified the ANKS1B protein to be part of a large post-synaptic complex, containing many neurotransmitter receptors and other proteins of relevance to schizophrenia (Fernandez et al, 2009). Clearly, the next step with $A N K S 1 B$, in addition to replication, is to identify possible causative variants. In our GWAS, we observed another ankyrin-related gene, ANKRD33 (ankyrin repeat domain 33), among the top findings. While there is currently little to no literature on this gene, its association in our GWAS may suggest a more general role for ankyrin proteins in antipsychotic response.

CHST8 (carbohydrate $\mathrm{N}$-acetylgalactosamine 4-0 sulfotransferase 8) was also significant at the genome-wide level. The function of its gene product is to carry out the sulfation of carbohydrates. Little published material is available on this gene, but it is considered a plausible pharmacokinetics candidate gene. For example, markers at this locus are included on the Affymetrix DMET plus array, which is designed to detect variation relevant to drug metabolizing enzymes and transporters (Burmester et al, 2010). The remaining genes among our top findings (ie, $q$-value $<$ 0.25 ) are either uncharacterized or have biological functions that do not immediately suggest obvious links to antipsychotic efficacy. For example, GPR137B (G proteincoupled receptor 137B), which achieved genome-wide significance for mediating the effects of olanzapine on working memory, is a member of the G protein-coupled receptor superfamily and is known to be expressed in brain (UniGene). However, its associated ligand has not yet been discovered and no other information on the function of this receptor is available currently. 
We present our results with some caveats and cautions. First, it has been shown previously that practice/placebo effects can be apparent with the types of neurocognitive outcome described here (Goldberg et al, 2007; Keefe et al, 2008). It is possible that the significant findings reported here reflect a genetically mediated ability to benefit from practice or general placebo effects such as expectation bias. Second, the reported effect sizes for the associated SNPs are most certainly overestimates. That is, because of sampling error, effect sizes in an initial study are often greater than those reported in subsequent replications (Goring et al, 2001; Ioannidis et al, 2001). Finally, chance findings are a possibility, as with any association study. Even with FDR control at the 0.1 level, 1 in 10 findings, on average, will be a false positive. This issue can only be addressed through replication in independent samples. To facilitate this, we provide all $p$-values (http://www.people.vcu.edu/ jlmcclay) for download as a resource for investigators with the requisite samples to carry out replication. However, to some extent, the GWAS would appear to have served its purpose, in that it immediately suggests novel follow-up experiments, in addition to simple replication.

\section{ACKNOWLEDGEMENTS}

The CATIE project was supported by NIMH contract N01 MH90001. Dr Sullivan was supported by R01s MH074027 and MH077139.

\section{DISCLOSURE}

Eli Lilly funded the GWAS genotyping performed at Perlegen Sciences. Dr Sullivan reports receiving research funding from Eli Lilly in connection with this project. Dr Stroup reports that in the past 3 years he has received consulting fees from Lilly and Janssen. Dr Lieberman reports having received research funding from AstraZeneca Pharmaceuticals, Bristol-Myers Squibb, GlaxoSmithKline, Janssen Pharmaceutica and Pfizer, and consulting and educational fees from AstraZeneca Pharmaceuticals, BristolMyers Squibb, Eli Lilly, Forest Pharmaceuticals, GlaxoSmithKline, Janssen Pharmaceutica, Novartis, Pfizer, and Solvay. Dr Keefe reports that he currently or in the past 12 months has received investigator-initiated research funding support from the National Institute of Mental Health, Allon, Novartis and the Singapore National Medical Research Council, and an unrestricted educational grant from AstraZeneca. He currently or in the past 12 months has received honoraria or served as a consultant or advisory board member for Abbott, Astra-Zeneca, BiolineRx, Bristol Myers Squibb, Cephalon, Dainippon Sumitomo Pharma, Eli Lilly, Johnson \& Johnson, Lundbeck, Memory Pharmaceuticals, Merck, Neurosearch, Orion, Orexigen, Otsuka, Pfizer, Roche, Targacept, Sanofi/Aventis, Shire, Wyeth, and Xenoport. In the past, he has received honoraria or served as a consultant or advisory board member for Acadia, Cortex, Cyberonics, Forest, Gabriel, GlaxoSmithKline, Repligen, Saegis, Shering-Plough, and has received research funding from Astra-Zeneca, Eli Lilly, Janssen, and Pfizer. Dr Keefe receives royalties from the Brief Assessment of Cognition in Schizophrenia (BACS) testing battery and the MATRICS
Battery (BACS Symbol Coding). Dr McEvoy declares receiving honoraria from Lilly, research grants and consulting fees from Sunovion, research grants from GlaxoSmithKline and Novartis, and serving on an advisory board with Merck. Drs McClay, Adkins, Åberg, Bukszár, Khachane, Perkins, Vann, Beardsley, and van den Oord declare no conflict of interest.

\section{REFERENCES}

Altshuler D, Daly MJ, Lander ES (2008). Genetic mapping in human disease. Science 322: 881-888.

Anttila S, Kampman O, Illi A, Rontu R, Lehtimaki T, Leinonen E (2007). Association between 5-HT2A, TPH1 and GNB3 genotypes and response to typical neuroleptics: a serotonergic approach. BMC Psychiatry 7: 22.

Arranz MJ, de Leon J (2007). Pharmacogenetics and pharmacogenomics of schizophrenia: a review of last decade of research. Mol Psychiatry 12: 707-747.

Banks WA, Farr SA, La Scola ME, Morley JE (2001). Intravenous human interleukin-1 $\alpha$ impairs memory processing in mice: dependence on blood-brain barrier transport into posterior division of the septum. J Pharmacol Exp Ther 299: 536-541.

Basile VS, Masellis M, Potkin SG, Kennedy JL (2002). Pharmacogenomics in schizophrenia: the quest for individualized therapy. Hum Mol Genet 11: 2517-2530.

Beecham GW, Martin ER, Li YJ, Slifer MA, Gilbert JR, Haines JL et al (2009). Genome-wide association study implicates a chromosome 12 risk locus for late-onset Alzheimer disease. Am J Hum Genet 84: 35-43.

Benjamini Y, Hochberg Y (1995). Controlling the false discovery rate: a practical and powerful approach to multiple testing. $J$ R Stat Soc B 57: 289-300.

Benton ALH, K (1978). Multilingual Aphasia Examination Manual (Revised). University of Iowa: Iowa City, IA.

Black MA (2004). A note on the adaptive control of false discovery rates. J R Stat Soc B 66: 297-304.

Brandt J (1991). The hopkins verbal learning test: development of a new memory test with six equivalent forms. Clin Neuropsychol 5: 125-142.

Brown BW, Russell K (1997). Methods of correcting for multiple testing: operating characteristics. Stat Med 16: 2511-2528.

Bukszar J, McClay JL, van den Oord EJ (2009). Estimating the posterior probability that genome-wide association findings are true or false. Bioinformatics 25: 1807-1813.

Burmester JK, Sedova M, Shapero MH, Mansfield E (2010). DMET microarray technology for pharmacogenomics-based personalized medicine. Methods Mol Biol 632: 99-124.

Campbell DB, Ebert PJ, Skelly T, Stroup TS, Lieberman J, Levitt P et al (2008). Ethnic stratification of the association of RGS4 variants with antipsychotic treatment response in schizophrenia. Biol Psychiatry 63: 32-41.

Cornblatt BA, Risch NJ, Faris G, Friedman D, Erlenmeyer-Kimling L (1988). The continuous performance test, identical pairs version (CPT-IP): I new findings about sustained attention in normal families. Psychiatry Res 26: 223-238.

Dorow DS, Devereux L, Dietzsch E, De Kretser T (1993). Identification of a new family of human epithelial protein kinases containing two leucine/isoleucine-zipper domains. Eur J Biochem 213: 701-710.

Dorwart MR, Shcheynikov N, Wang Y, Stippec S, Muallem S (2007). SLC26A9 is a $\mathrm{Cl}(-)$ channel regulated by the WNK kinases. J Physiol 584(Pt 1): 333-345.

Efron BT, Storey R, Tusher JD, V (2001). Empirical Bayes analysis of a microarray experiment. J Am Stat Assoc 96: 1151-1160. 
Fernandez E, Collins MO, Uren RT, Kopanitsa MV, Komiyama NH, Croning MD et al (2009). Targeted tandem affinity purification of PSD-95 recovers core postsynaptic complexes and schizophrenia susceptibility proteins. Mol Syst Biol 5: 269.

First MS, Gibbon R, Williams M, J (1994). Structured Clinical Interview for DSM-IV Axis I Disorders-Administration Booklet. American Psychiatric Press: Washington DC.

Garriock HA, Kraft JB, Shyn SI, Peters EJ, Yokoyama JS, Jenkins GD et al (2010). A genomewide association study of citalopram response in major depressive disorder. Biol Psychiatry 67: 133-138.

Gold JM, Carpenter C, Randolph C, Goldberg TE, Weinberger DR (1997). Auditory working memory and wisconsin card sorting test performance in schizophrenia. Arch Gen Psychiatry 54: $159-165$.

Goldberg TE, Goldman RS, Burdick KE, Malhotra AK, Lencz T, Patel RC et al (2007). Cognitive improvement after treatment with second-generation antipsychotic medications in firstepisode schizophrenia: is it a practice effect? Arch Gen Psychiatry 64: $1115-1122$.

Goldstein H (1995). Multilevel Statistical Models. Arnold: London.

Goring HH, Terwilliger JD, Blangero J (2001). Large upward bias in estimation of locus-specific effects from genomewide scans. Am J Hum Genet 69: 1357-1369.

Green MF (1996). What are the functional consequences of neurocognitive deficits in schizophrenia? Am J Psychiatry 153: 321-330.

Greenwood TA, Braff DL, Light GA, Cadenhead KS, Calkins ME, Dobie DJ et al (2007). Initial heritability analyses of endophenotypic measures for schizophrenia: the consortium on the genetics of schizophrenia. Arch Gen Psychiatry 64: 1242-1250.

Hadi AS, Simonoff JS (1993). Procedures for the identification of multiple outliers in linear-models. J Am Stat Assoc 88: 1264-1272.

Hamdani N, Tabeze JP, Ramoz N, Ades J, Hamon M, Sarfati Y et al (2008). The CNR1 gene as a pharmacogenetic factor for antipsychotics rather than a susceptibility gene for schizophrenia. Eur Neuropsychopharmacol 18: 34-40.

He G, Wang HR, Huang SK, Huang CL (2007). Intersectin links WNK kinases to endocytosis of ROMK1. J Clin Invest 117: 1078-1087.

Heaton RK, Chelune GJ, Taley JL, Kay GG, Curtiss G (1993). Wisconsin Card Sorting Test Manual: Revised and Expanded. Psychological Assessment Resources: Odessa, FL.

Hill SK, Harris MS, Herbener ES, Pavuluri M, Sweeney JA (2008). Neurocognitive allied phenotypes for schizophrenia and bipolar disorder. Schizophr Bull 34: 743-759.

Hwang R, Shinkai T, De Luca V, Ni X, Potkin SG, Lieberman JA et al (2007). Association study of four dopamine D1 receptor gene polymorphisms and clozapine treatment response. J Psychopharmacol 21: 718-727.

Hyman SE, Fenton WS (2003). Medicine. What are the right targets for psychopharmacology? Science 299: 350-351.

Ioannidis JP, Ntzani EE, Trikalinos TA, Contopoulos-Ioannidis DG (2001). Replication validity of genetic association studies. Nat Genet 29: 306-309.

Kahle KT, Macgregor GG, Wilson FH, Van Hoek AN, Brown D, Ardito $\mathrm{T}$ et al (2004). Paracellular Cl- permeability is regulated by WNK4 kinase: insight into normal physiology and hypertension. Proc Natl Acad Sci USA 101: 14877-14882.

Kane JM (1999). Pharmacologic treatment of schizophrenia. Biol Psychiatry 46: 1396-1408.

Kapur S, Zipursky RB, Remington G (1999). Clinical and theoretical implications of 5-HT2 and D2 receptor occupancy of clozapine, risperidone, and olanzapine in schizophrenia. Am J Psychiatry 156: 286-293.

Kas K, Finger E, Grall F, Gu X, Akbarali Y, Boltax J et al (2000). ESE-3, a novel member of an epithelium-specific ets transcription factor subfamily, demonstrates different target gene specificity from ESE-1. J Biol Chem 275: 2986-2998.

Keefe RS, Bilder RM, Davis SM, Harvey PD, Palmer BW, Gold JM et al (2007a). Neurocognitive effects of antipsychotic medications in patients with chronic schizophrenia in the CATIE Trial. Arch Gen Psychiatry 64: 633-647.

Keefe RS, Bilder RM, Harvey PD, Davis SM, Palmer BW, Gold JM et al (2006). Baseline neurocognitive deficits in the CATIE schizophrenia trial. Neuropsychopharmacology 31: 2033-2046.

Keefe RS, Malhotra AK, Meltzer HY, Kane JM, Buchanan RW, Murthy A et al (2008). Efficacy and safety of donepezil in patients with schizophrenia or schizoaffective disorder: significant placebo/practice effects in a 12-week, randomized, doubleblind, placebo-controlled trial. Neuropsychopharmacology 33: 1217-1228.

Keefe RS, Mohs RC, Bilder RM, Harvey PD, Green MF, Meltzer HY et al (2003). Neurocognitive assessment in the Clinical Antipsychotic Trials of Intervention Effectiveness (CATIE) project schizophrenia trial: development, methodology, and rationale. Schizophr Bull 29: 45-55.

Keefe RS, Sweeney JA, Gu H, Hamer RM, Perkins DO, McEvoy JP et al (2007b). Effects of olanzapine, quetiapine, and risperidone on neurocognitive function in early psychosis: a randomized, double-blind 52-week comparison. Am J Psychiatry 164: 1061-1071.

Kirchheiner J, Nickchen K, Bauer M, Wong ML, Licinio J, Roots I et al (2004). Pharmacogenetics of antidepressants and antipsychotics: the contribution of allelic variations to the phenotype of drug response. Mol Psychiatry 9: 442-473.

Kremen WS, Seidman LJ, Faraone SV, Toomey R, Tsuang MT (2004). Heterogeneity of schizophrenia: a study of individual neuropsychological profiles. Schizophr Res 71: 307-321.

Lafayette Instrument Company (1989). Grooved Pegboard Instruction Manual (Model 32025). Lafayette Instrument Company: Lafayette, IN.

Lei SF, Yang TL, Tan LJ, Chen XD, Guo Y, Guo YF et al (2009). Genome-wide association scan for stature in Chinese: evidence for ethnic specific loci. Hum Genet 125: 1-9.

Lieberman JA, Stroup TS, McEvoy JP, Swartz MS, Rosenheck RA, Perkins DO et al (2005). Effectiveness of antipsychotic drugs in patients with chronic schizophrenia. $N$ Engl J Med 353: $1209-1223$.

Liu YZ, Guo YF, Wang L, Tan LJ, Liu XG, Pei YF et al (2009). Genome-wide association analyses identify SPOCK as a key novel gene underlying age at menarche. PLoS Genet 5: e1000420.

Loriol C, Dulong S, Avella M, Gabillat N, Boulukos K, Borgese F et al (2008). Characterization of SLC26A9, facilitation of $\mathrm{Cl}(-)$ transport by bicarbonate. Cell Physiol Biochem 22: 15-30.

Lyons-Warren A, Lillie R, Hershey T (2004). Short- and long-term spatial delayed response performance across the lifespan. Dev Neuropsychol 26: 661-678.

Malhotra AK, Murphy Jr GM., Kennedy JL (2004). Pharmacogenetics of psychotropic drug response. Am J Psychiatry 161: 780-796.

Mancama D, Mata I, Kerwin RW, Arranz MJ (2007). Choline acetyltransferase variants and their influence in schizophrenia and olanzapine response. Am J Med Genet B Neuropsychiatr Genet 144B: 849-853.

McClay JL, Adkins DE, Aberg K, Stroup S, Perkins DO, Vladimirov VI et al (2009). Genome-wide pharmacogenomic analysis of response to treatment with antipsychotics. Mol Psychiatry; e-pub ahead of print 1 September 2009, PMID 19721433.

Meary A, Brousse G, Jamain S, Schmitt A, Szoke A, Schurhoff F et al (2008). Pharmacogenetic study of atypical antipsychotic drug response: involvement of the norepinephrine transporter gene. Am J Med Genet B Neuropsychiatr Genet 147B: 491-494.

Meisenzahl EM, Rujescu D, Kirner A, Giegling I, Kathmann N, Leinsinger $\mathrm{G}$ et al (2001). Association of an interleukin-1beta 
genetic polymorphism with altered brain structure in patients with schizophrenia. Am J Psychiatry 158: 1316-1319.

Meltzer HY, Okayli G (1995). Reduction of suicidality during clozapine treatment of neuroleptic-resistant schizophrenia: impact on risk-benefit assessment. Am J Psychiatry 152: 183-190.

Mesholam-Gately RI, Giuliano AJ, Goff KP, Faraone SV, Seidman LJ (2009). Neurocognition in first-episode schizophrenia: a meta-analytic review. Neuropsychology 23: 315-336.

Need AC, Keefe RS, Ge D, Grossman I, Dickson S, McEvoy JP et al (2009). Pharmacogenetics of antipsychotic response in the CATIE trial: a candidate gene analysis. Eur J Hum Genet 17: 946-957.

O’Donovan MC, Craddock N, Norton N, Williams H, Peirce T, Moskvina $\mathrm{V}$ et al (2008). Identification of loci associated with schizophrenia by genome-wide association and follow-up. Nat Genet 40: 1053-1055.

Pinheiro JC, Bates DM (2000). Mixed-Effects Models in S and SPlus. Springer: New York, NY.

Purcell S, Neale B, Todd-Brown K, Thomas L, Ferreira MA, Bender D et al (2007). PLINK: a tool set for whole-genome association and population-based linkage analyses. Am J Hum Genet 81: 559-575.

Roses AD (2000). Pharmacogenetics and the practice of medicine. Nature 405: 857-865.

Sanger DJ (2004). The search for novel antipsychotics: pharmacological and molecular targets. Expert Opin Ther Targets 8: 631-641.

Searle SR, Casella G, McCulloch CE (1992). Variance Components. Wiley: New York, NY.

Shi J, Levinson DF, Duan J, Sanders AR, Zheng Y, Pe'er I et al (2009). Common variants on chromosome 6p22.1 are associated with schizophrenia. Nature 460: 753-757.

Storey J (2003). The positive false discovery rate: a Bayesian interpretation and the q-value. Ann Stat 31: 2013-2035.

Storey JD, Tibshirani R (2003). Statistical significance for genomewide studies. Proc Natl Acad Sci USA 100: 9440-9445.

Stroup TS, McEvoy JP, Swartz MS, Byerly MJ, Glick ID, Canive JM et al (2003). The National Institute of Mental Health Clinical
Antipsychotic Trials of Intervention Effectiveness (CATIE) project: schizophrenia trial design and protocol development. Schizophr Bull 29: 15-31.

Sullivan PF, Lin D, Tzeng JY, van den Oord E, Perkins D, Stroup TS et al (2008). Genomewide association for schizophrenia in the CATIE study: results of stage 1. Mol Psychiatry 13: 570-584.

Thierry-Mieg D, Thierry-Mieg J (2006). AceView: a comprehensive cDNA-supported gene and transcripts annotation. Genome Biol 7(Suppl 1): 11-S12 14.

Tsukita S, Furuse M (2000). Pores in the wall: claudins constitute tight junction strands containing aqueous pores. J Cell Biol 149: 13-16.

Tugores A, Le J, Sorokina I, Snijders AJ, Duyao M, Reddy PS et al (2001). The epithelium-specific ETS protein EHF/ESE-3 is a context-dependent transcriptional repressor downstream of MAPK signaling cascades. J Biol Chem 276: 20397-20406.

van den Oord EJ, Adkins DE, McClay J, Lieberman J, Sullivan PF (2009). A systematic method for estimating individual responses to treatment with antipsychotics in CATIE. Schizophr Res 107: 13-21.

van den Oord EJ, Sullivan PF (2003). False discoveries and models for gene discovery. Trends Genet 19: 537-542.

Wechsler D (1981). WAIS-R Manual: Wechsler Adult Intelligence Scale-Revised. Psychological Corporation: New York, NY.

Wechsler D (1991). Wechsler Intelligence Scale for Children. Psychological Corporation: San Antonio, TX.

Wheeler DL, Church DM, Federhen S, Lash AE, Madden TL, Pontius JU et al (2003). Database resources of the National Center for Biotechnology. Nucleic Acids Res 31: 28-33.

Williams M (2003). Genome-based drug discovery: prioritizing disease-susceptibility/disease-associated genes as novel drug targets for schizophrenia. Curr Opin Investig Drugs 4: 31-36.

Zandi PP, Judy JT (2010). The promise and reality of pharmacogenetics in psychiatry. Psychiatr Clin North Am 33: 181-224.

Zaykin DV, Young SS, Westfall PH (2000). Using the false discovery rate approach in the genetic dissection of complex traits: a response to Weller et al. Genetics 154: 1917-1918.

Supplementary Information accompanies the paper on the Neuropsychopharmacology website (http://www.nature.com/npp) 